\title{
p40 Immunohistochemistry Is an Excellent Marker in Primary Lung Squamous Cell Carcinoma
}

\begin{abstract}
Khairunisa Ahmad Affandi
Nur Maya Sabrina Tizen

Muaatamarulain Mustangin

Reena Rahayu Md Zin

Department of Pathology, Universiti Kebangsaan Malaysia Medical Centre, Universiti Kebangsaan Malaysia, Kuala Lumpur, Malaysia
\end{abstract}

\author{
Received: April 23, 2018 \\ Revised: August 9, 2018 \\ Accepted: August 14, 2018 \\ Corresponding Author \\ Reena Rahayu Md Zin, MBChB, PhD \\ Department of Pathology, Faculty of Medicine, UKM \\ Medical Center, Jalan Yaacob Latif, Bandar Tun \\ Razak, Cheras 56000, Kuala Lumpur, Malaysia \\ Tel: +603-91455359 \\ Fax: +603-91737340 \\ E-mail: reenarahayu@ppukm.ukm.edu.my
}

\begin{abstract}
Background: Lung cancer is the third most common cancer worldwide. With major advances in the molecular testing of lung cancers and the introduction of targeted therapies, the distinction between adenocarcinoma and squamous cell carcinoma as well as pathologic subtyping has become important. Recent studies showed that p40 is highly specific for squamous and basal cells and is superior to p63 for diagnosing lung squamous cell carcinoma. The aim of this study was to evaluate the use of p40 immunohistochemical stain in the diagnosis of non-small cell lung carcinoma and its potential to replace current p63 antibody as the best immunohistochemical squamous marker. Methods: Seventy formalin-fixed paraffin-embedded cases previously diagnosed as primary lung squamous cell carcinoma $(n=35)$ and lung adenocarcinoma $(n=35)$ from January 2008 to December 2016 were retrieved. The results of tumour cell immunoreactivity for p40 and p63 antibodies in lung squamous cell carcinoma and lung adenocarcinoma were compared. Results: p40 was expressed in 27 cases of lung squamous cell carcinoma (77.1\%). All cases of lung adenocarcinoma $(35 / 35,100 \%)$ were negative for p40. p63 expression was positive in 30 cases of lung squamous cell carcinoma $(85.7 \%)$ and 13 cases of lung adenocarcinoma (37.1\%). Reactivity for both p40 and p63 in lung squamous cell carcinoma was strong and diffuse, whereas variable reactivity was observed in lung adenocarcinoma. Conclusions: p40 is an excellent marker for distinguishing lung squamous cell carcinoma from adenocarcinoma, and p40 expression is equivalent to p63 expression in lung squamous cell carcinoma.
\end{abstract}

Key Words: Non-small cell lung carcinoma; Immunohistochemistry; Lung neoplasms; p40; p63
Lung cancer is the most common cancer among males and the third most common cancer in the general population of Malaysia as well as worldwide. Worldwide, there was an estimated 1.8 million new cases of lung cancer in 2012. ${ }^{1}$ In Malaysia, there were 10,608 cases of lung cancer diagnosed between 2007 to 2011, comprising 7,415 males and 3,193 females. ${ }^{2}$ Lung cancer is also the most common cause of cancer deaths and is estimated to be responsible for 1.59 million mortalities. ${ }^{1}$ Most lung cancers are detected at a late stage, and $60 \%$ of cases were detected at stage IV. ${ }^{1}$

With recent advances in molecular testing of lung cancers and the introduction of targeted therapies, distinction between adenocarcinoma and squamous cell carcinoma, as well as pathologic subtyping, has become increasingly important. Specific therapies can be offered to patients depending on the histologic diagnosis and molecular status of the tumour. For example, patients with lung adenocarcinoma harbouring epidermal growth factor receptor (EGFR) mutations and anaplastic lymphoma kinase $(A L K)$ rearrangement are given first-line treat- ment with tyrosine kinase inhibitors and crizotinib, respectively, while patients with squamous cell carcinoma are contraindicated for bevacizumab therapy, as it is associated with life-threatening haemorrhage. ${ }^{3,4}$ Therefore, it is crucial to establish the accurate histologic subtype to guide patient selection for further molecular testing.

Patients with advanced disease are usually inoperable, so they often receive targeted molecular therapies. ${ }^{3}$ In such situations, a small biopsy and cytology preparations are often the only specimens available for histologic diagnosis and molecular testing. ${ }^{3}$ This has led to major changes in the latest pathologic classification by the World Health Organization (WHO) with particular emphasis on tumour classification and strategic tissue management for handling non-resected specimens. ${ }^{4}$ The diagnosis of adenocarcinoma and squamous cell carcinoma can be made based on morphology alone in $50 \%-70 \%$ of non-resected specimens. ${ }^{4}$ However, immunohistochemistry and special stains are often required in the setting of poorly differentiated tumours that do not show definite morphology by routine light microscopy. 
The recommended markers for adenocarcinoma are thyroid transcription factor 1 (TTF1) and napsin A, whereas accepted antibodies for squamous differentiation include p63, p40, and cytokeratin (CK) 5/6. ${ }^{4}$ To preserve as much tissue as possible for molecular studies, algorithms recommend a maximum use of two antibodies in each case. ${ }^{4,6,7}$ Most tumours can be classified using a single adenocarcinoma marker and a single squamous marker., ${ }^{4,6}$

p63 is a homologue of the p53 tumour suppressor gene that is responsible for proliferation and differentiation of epithelial progenitor cells. ${ }^{8}$ The 553 gene contains two promoters that produce two isoforms; one isoform contains the $\mathrm{N}$-terminal transactivation domain (TAp63) and the other lacks this domain $(\triangle \mathrm{Np} 63){ }^{8} \mathrm{p} 63$ is normally expressed in the nuclei of basal and progenitor cells of stratified epithelia such as skin, esophagus, tonsil, urothelium, ectocervix, and vagina, and in the basal cells of glandular structures of the thymus, prostate, breast, and bronchi. ${ }^{8}$ Both TAp63 and $\triangle \mathrm{Np} 63$ show overlapping distribution in some epithelial tissue. However, TAp63 is more expressed in differentiated cells while $\Delta \mathrm{Np} 63$ is seen in the stem-like cell populations. ${ }^{8}$

Anti-p63 (4A4) is a well-accepted immunohistochemical marker for lung squamous cell carcinoma in most laboratories. Despite having extremely high sensitivity, as antibodies for p63 (4A4) recognize both p63 and p40 proteins, studies have shown it has a lack of specificity in a subset of lung cancers, particularly lung adenocarcinoma. ${ }^{4,9}$ Focal or weak p63 staining can be seen in $30 \%$ of lung adenocarcinoma and has been misinterpreted to favour squamous differentiation. ${ }^{3,10}$ Interpretation of $\mathrm{p} 63$ is also dependent on TTF1 expression because only diffuse p63 staining in the absence of TTF1 is considered to favour squamous cell carcinoma. ${ }^{4}$ An incorrect diagnosis of squamous cell carcinoma could be made with unforeseen faulty TTF1 immunostaining. ${ }^{8}$ Moreover, additional squamous markers such as $\mathrm{CK} 5 / 6$ may be required, which compromises tissue preservation for molecular studies. ${ }^{8,11}$

Antibody p 40 , which identifies $\Delta \mathrm{Np} 63$, has been available for several years but its use for distinction of lung squamous cell carcinoma and adenocarcinoma was only recently studied. p40 is consistently the predominant isoform expressed in squamous cell carcinoma; thus, it offers improved specificity for diagnosing squamous cell carcinoma. ${ }^{8}$ Studies by Bishop et al. ${ }^{9}$ and Nonaka ${ }^{10}$ showed that p 40 has $100 \%$ sensitivity and specificity in lung squamous cell carcinoma. Another study by Tacha et al..$^{12}$ reported an $85 \%$ sensitivity and $98 \%$ specificity.

In local Malaysian diagnostic histopathology laboratories, selection of immunohistochemistry studies is highly dependent on the morphologic features present on hematoxylin and eosin sections and the availability of markers. Currently, there is no standardized diagnostic immunohistochemical workup in the diagnosis of non-small cell lung carcinoma (NSCLC). However, a panel of TTF1, CK5 $/ 6$, and p63 is the most commonly used set of immunohistochemistry studies for distinguishing adenocarcinoma from squamous cell carcinoma.

In this study, we investigated the expression of $\mathrm{p} 40$ in lung squamous cell carcinoma and lung adenocarcinoma in comparison to p63 expression. In addition, the present study was performed to evaluate the utility of p40 and p63 in arriving at an accurate diagnosis in NSCLC.

\section{MATERIALS AND METHODS}

This study was approved by the Ethical Committee of Universiti Kebangsaan Malaysia Ref No. UKM 1.5.3.5/244/JEP2016-089 and was exempted from informed consent by the institutional review board (IRB). All cases diagnosed as primary lung squamous cell carcinoma and adenocarcinoma between January 2008 and December 2016 were reviewed. Cases that fulfilled the inclusion and exclusion criteria with sufficient tissue samples were selected for this study. Hematoxylin and eosin stained slides were reviewed by two pathologists to confirm the diagnosis. Tumours were graded on the basis of WHO classification. The tissues were obtained from routine formalin-fixed paraffin-embedded blocks of small biopsies and resected specimens of 35 lung squamous cell carcinoma and 35 lung adenocarcinoma cases. Information on patient details was retrieved from the Computerized Medical Record System. Patient identity remained anonymous and each subject was coded accordingly.

\section{Primary antibodies}

Two antibodies were used in this study, mouse monoclonal p63 antibody (Clone DAK-p63, Ready-To-Use, Code IR662, Dako, Glostrup, Denmark) and mouse monoclonal p40 antibody (1:100, clone BC28, Code Ab172731, Abcam, Cambridge, UK). Normal skin and laryngeal squamous cell carcinoma tissues were used as the positive controls for p63 and p40, respectively.

\section{Immunohistochemical staining method}

Immunohistochemical staining was performed on tissue sections using the protocol from EnVision FLEX+, Mouse, High pH (Code No. K8012, Dako). Primary antibody was diluted to 
optimal concentration using Antibody Diluent, Dako REAL (Code No. S2022, Dako).

The tissue blocks were sectioned to approximately $3 \mu \mathrm{m}$ thickness. The tissue slides were incubated on hot plates at $60^{\circ} \mathrm{C}$ for 1 hour and initial deparaffinization and pre-treatment was performed in the Decloaking Chamber NxGen (Ref. No: DC2012-220V, Biocare Medical, Pacheco, CA, USA) using EnVision FLEX Target Retrieval Solution, High pH (Code No. $\mathrm{DM} 828, \mathrm{Dak}$ ) at $110^{\circ} \mathrm{C}$ for 30 minutes. Slides were incubated with the primary antibodies for 20 minutes at room temperature, followed by incubation with EnVision FLEX/HRP (Code No. DM822, Dako) for 20 minutes. Sections were then incubated with $1 \times$ DAB-containing Substrate Working Solution for 10 minutes. Slides were then counterstained with Hematoxylin 2 (REF 7231, Thermo Scientific, Rockford, IL, USA).

\section{Immunohistochemical staining analysis}

The staining intensity of both p40 and p63 markers were scored as $0,1+, 2+$, or $3+$ (Fig. 1 ) and the percentage of immunoreactive cells were recorded. ${ }^{9}$ Cases were considered positive if $5 \%$ or more of the tumour cells showed brown nuclear staining. Cases with less than $5 \%$ staining or no areas of positive staining were regarded as negative.

\section{Statistical analysis}

Data analysis was performed using the SPSS software program ver. 20.0 (IBM Corp., Armonk, NY, USA). Pearson chisquare test was used to assess the association between positivity of each marker and tumour diagnosis. Here, p-values less than .05 were considered statistically significant.

\section{RESULTS}

\section{Epidemiologic and clinico pathologic data}

A total of 70 cases of NSCLC were investigated. More than half of the cases presented with distant metastasis at diagnosis (64.2\%). For squamous cell carcinoma, 32 (91.4\%) cases were males and three cases $(8.6 \%)$ were females. The mean age at squamous cell carcinoma diagnosis was 68 years, ranging from 36 to 98 years. Adenocarcinoma had a more similar sex distribution, with 19 cases $(54.3 \%)$ in males and 16 cases $(45.7 \%)$ in females. The mean age for adenocarcinoma was 59 years, ranging from 25 to 90 years. Squamous cell carcinomas comprised seven well, 11 moderately, and 15 poorly differentiated tumours. Among the adenocarcinomas, there were nine well, 14 moderately, and 12 poorly differentiated carcinomas. The clinical information is summarised in Table 1.

\section{p40 immunostaining}

Twenty-seven cases of primary lung squamous cell carcinoma (77.1\%) were positive for p40 (Table 2). The reactivity of p40 nuclear staining in lung squamous cell carcinoma was strong and diffuse; $71.4 \%$ of cases had intensity score of $3+$ in more than $50 \%$ of tumour cells (Table 3, Fig. 2). Remarkably, eight cases that were previously diagnosed as primary lung squamous cell carcinoma showed negative staining. Of these, six cases $(17.1 \%)$ had intensity score of 0 and two cases $(5.7 \%)$ showed minimal reactivity (less than $5 \%$ of tumour cells) with intensity score of $1+$. The original slides were reviewed and all of these cases are poorly differentiated tumours with focal squamouslike morphology including solid growth pattern, abundant eosinophilic cytoplasm (resembling cytoplasmic keratinization), sharp cell borders, and intercellular bridges (Fig. 2). Six cases
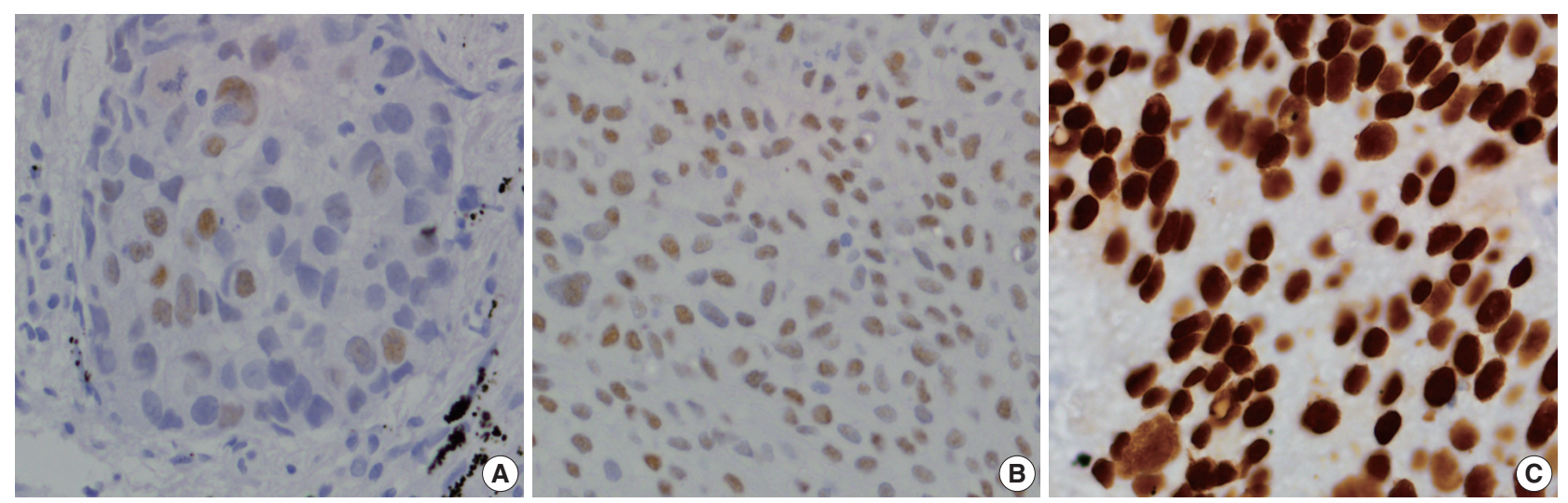

Fig. 1. Intensity score for positive p40 and p63 nuclear staining in tumour cells. (A) Score 1+ for weak positivity. (B) Score 2+ for moderate positivity. (C) Score 3+ for strong positivity. 
had prior immunohistochemistry and the diagnosis of squamous cell carcinoma was made on the basis of negative TTF1 and $\mathrm{p} 63$ positivity $(\mathrm{n}=3)$. The other two cases were diagnosed as squamous cell carcinoma based on morphology alone. In con-

Table 1. Demographic and clinicopathologic data of patients diagnosed with lung squamous cell carcinoma and lung adenocarcinoma

\begin{tabular}{lcc}
\hline & $\begin{array}{c}\text { Squamous cell } \\
\text { carcinoma }\end{array}$ & Adenocarcinoma \\
\hline Sex, $\mathrm{n}(\%)$ & $32(91.4)$ & $19(54.3)$ \\
Male & $3(8.6)$ & $16(45.7)$ \\
Female & $68 \pm 12.8$ & $59 \pm 14.2$ \\
Age at diagnosis, mean \pm SD (yr) & & \\
Ethnicity & 23 & 20 \\
Malay & 11 & 14 \\
Chinese & 0 & 0 \\
Indian & 1 & 1 \\
Others & 7 & 9 \\
Histologic grade & 12 & 14 \\
Well differentiated & 16 & \\
Moderately differentiated & & \\
\hline Poorly differentiated & & \\
\hline
\end{tabular}

SD, standard deviation. trast, all (100\%) lung adenocarcinomas were negative for $\mathrm{p} 40$.

Two cases of lung adenocarcinoma showed weak p40 expression $(1+)$. However, the immunoreactive cells were minimal (in less than $5 \%$ of tumour cells) and scattered with no specific pattern. Immunopositivity of p40 and p63 according to tumour differentiation is shown in Table 4.

Table 5 summarizes immunohistochemical profile of $\mathrm{p} 40$ negative squamous cell carcinoma and histologic grading.

\section{p63 immunostaining}

Thirty cases $(85.7 \%)$ of lung squamous cell carcinoma were

Table 2. Expression of p40 and p63 in lung squamous cell carcinoma and lung adenocarcinoma

\begin{tabular}{ccc}
\hline & Squamous cell carcinoma & Adenocarcinoma \\
\hline p40 & & \\
Positive & $27(77.1)$ & 0 \\
Negative & $8(22.9)$ & $35(100)$ \\
p63 & & \\
Positive & $30(85.7)$ & $13(37.1)$ \\
Negative & $5(14.3)$ & $22(62.9)$ \\
\hline
\end{tabular}

Values are presented as number (\%).

Table 3. Immunoreactivity for p40 and p63 in lung SCC and lung ADC

\begin{tabular}{|c|c|c|c|c|c|c|c|c|c|}
\hline & \multirow{2}{*}{ No. } & \multicolumn{4}{|c|}{ Cases with the following intensity score } & \multicolumn{4}{|c|}{ Cases with the following proportion of immunoreactive cells } \\
\hline & & 0 & 1 & 2 & 3 & $0 \%-4 \%$ & $5 \%-25 \%$ & $26 \%-50 \%$ & $>50 \%$ \\
\hline \multicolumn{10}{|l|}{ SCC } \\
\hline $\mathrm{p} 40$ & 35 & $6(17.1)$ & $3(8.6)$ & $1(2.9)$ & $25(71.4)$ & $2(5.7)$ & $2(5.7)$ & 0 & $25(71.4)$ \\
\hline p63 & 35 & $4(11.4)$ & $2(5.7)$ & $3(8.6)$ & $26(74.3)$ & $1(2.9)$ & $4(11.4)$ & 0 & $26(74.3)$ \\
\hline \multicolumn{10}{|l|}{ ADC } \\
\hline $\mathrm{p} 40$ & 35 & $33(94.3)$ & $2(5.7)$ & 0 & 0 & $2(5.7)$ & 0 & 0 & 0 \\
\hline p63 & 35 & $13(37.1)$ & $9(25.7)$ & $8(22.9)$ & $5(14.3)$ & $9(25.7)$ & $6(17.1)$ & $5(14.3)$ & $2(5.7)$ \\
\hline
\end{tabular}

Values are presented as number (\%).

SCC, squamous cell carcinoma; ADC, adenocarcinoma.

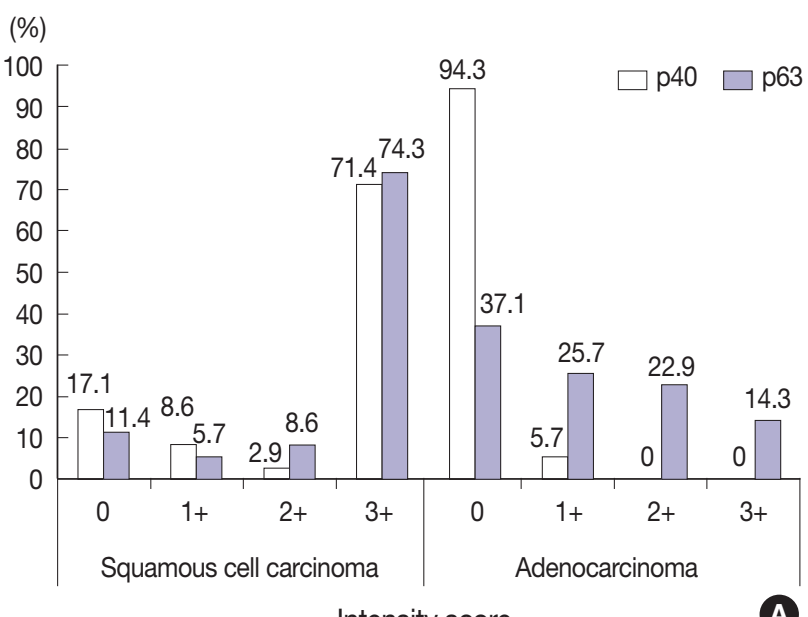

Intensity score

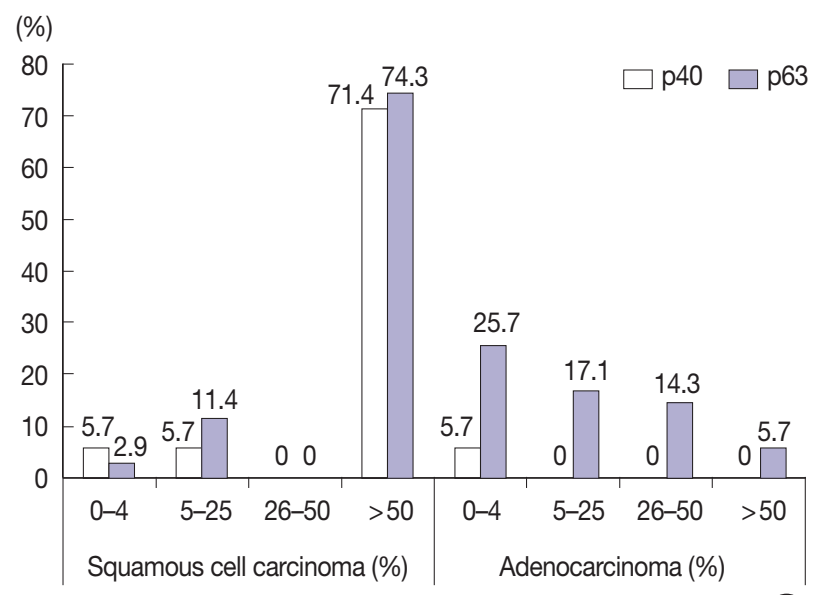

Tumour cell immunoreactivity

Fig. 2. Analysis of p40 and p63 expression in lung squamous cell carcinoma and adenocarcinoma. (A) Intensity score. (B) Tumour cell immunoreactivity. 
positive for p63 (Table 2). The expression of p63 in lung squamous cell carcinoma was also strong and diffuse, equivalent to that of $\mathrm{p} 40$, and $74.3 \%$ of cases had intensity score of $3+$ in more than $50 \%$ of tumour cells. Among lung adenocarcinomas, 13 cases $(37.1 \%)$ showed p63 positivity. These cases demonstrated a vast range of immunoreactivity, with minimal reactivity (less than $5 \%$ of tumour cells) in nine cases, focal reactivity ( 5 to $50 \%$ of tumour cells) in 11 cases, and diffuse reactivity (more than $50 \%$ of tumour cells) in two cases (Table 3, Fig. 2).

All $\mathrm{p} 63$ negative cases were also negative for $\mathrm{p} 40$. Conversely, three $\mathrm{p} 40$ negative cases were $\mathrm{p} 63$ positive (Table 5). In their benign counterparts, both $\mathrm{p} 40$ and $\mathrm{p} 63$ showed nuclear staining in basal bronchial cells.

As shown in Table 6, the specificity of $\mathrm{p} 40$ for lung squamous cell carcinoma was $100 \%$ compared to $62.9 \%$ for p63. The sensitivity of p 40 and $\mathrm{p} 63$ was $77.1 \%$ and $85.7 \%$, respec-

Table 4. Immunopositivity of p40 and p63 according to tumour differentiation

\begin{tabular}{lrrr}
\hline Tumour differentiation & No. & p40 & \multicolumn{1}{c}{ p63 } \\
\hline $\begin{array}{l}\text { Squamous cell carcinoma } \\
\text { Well differentiated }\end{array}$ & 7 & $7 / 7(100)$ & $7 / 7(100)$ \\
$\quad$ Moderately differentiated & 12 & $12 / 12(100)$ & $12 / 12(100)$ \\
$\quad$ Poorly differentiated & 16 & $8 / 16(50)$ & $11 / 16(68.8)$ \\
Adenocarcinoma & & & \\
Well differentiated & 9 & $0 / 9(0)$ & $3 / 9(33.3)$ \\
$\quad$ Moderately differentiated & 14 & $0 / 14(0)$ & $8 / 14(57.1)$ \\
Poorly differentiated & 12 & $0 / 12(0)$ & $2 / 12(16.7)$ \\
\hline
\end{tabular}

Values are presented as number (\%). tively. p40 has a higher positive predictive value for lung squamous cell carcinoma than p63 (100\% vs $69.8 \%)$. Both markers had similar negative predictive values.

\section{DISCUSSION}

NSCLC, constituting tumours other than small cell carcinoma, accounts for $85 \%$ of all lung cancers; lung adenocarcinoma and squamous cell carcinoma are the most frequent histologic subtypes. ${ }^{4,13}$ Morphologically, adenocarcinoma is identified by glandular differentiation and mucin production. Squamous differentiation is recognized by keratinization, keratin pearls, and intercellular bridges. ${ }^{4}$ However, distinguishing between the two can be challenging, particularly in poorly differentiated tumours. In such cases, immunohistochemical markers are recommended for precise histologic subtyping. ${ }^{4}$ To date, the WHO recommends that cases should be classified as NSCLC, favouring adenocarcinoma when they are positive for an adenocarcinoma marker (TTF1 or mucin) with a negative squamous marker (p63 or p40). Conversely, cases with at least moderate diffuse staining for a squamous marker and negative adenocarcinoma marker should be classified as NSCLC, favouring squamous cell carcinoma.

Although most lung tumours can be accurately subtyped by co-expression of TTF1 and p63, a small percentage of cases showed overlapping (TTF1+/p63+) or indeterminate staining patterns (TTF1/weak p63+), leading to misinterpretation and

Table 5. Histologic findings and IHC profile of p40-negative lung squamous cell carcinoma

\begin{tabular}{|c|c|c|c|c|c|c|}
\hline Case No. & Nature of specimen & p40 & p63 & Histologic grade & Squamoid morphology & Other $I_{\mathrm{HC}}$ \\
\hline 2 & Lung biopsy & $\begin{array}{c}1+ \\
\text { (in }<5 \%)\end{array}$ & $1+$ & Poorly differentiated & - & $\mathrm{CK} 7+, \mathrm{TTF} 1-$ \\
\hline 3 & Lung biopsy & 0 & 0 & Poorly differentiated & Focal intercellular bridging and keratinization & - \\
\hline 4 & Lung biopsy & 0 & 0 & Poorly differentiated & Intercellular bridging and keratinization & - \\
\hline 9 & Lung biopsy & 0 & $\begin{array}{c}2+ \\
\text { (in }>50 \%)\end{array}$ & Poorly differentiated & Abundant eosinophilic cytoplasm & $\mathrm{CK}+, \mathrm{TF} 1+$ (focal) \\
\hline 21 & Lung biopsy & 0 & $\begin{array}{c}2+ \\
\text { (in } 5 \%-25 \%)\end{array}$ & Poorly differentiated & Focal intercellular bridging and keratinization & TTF1-, p63+ \\
\hline 22 & Lung biopsy & 0 & 0 & Poorly differentiated & Abundant eosinophilic cytoplasm & TTF1-, CK5/6- \\
\hline 33 & Lung biopsy & 0 & 0 & Poorly differentiated & Abundant eosinophilic cytoplasm & $\begin{array}{l}\text { CK7+ (focal), } \\
\text { p63+ (focal), TTF1- }\end{array}$ \\
\hline 34 & Lung biopsy & $\begin{array}{c}1+ \\
\text { (in }<5 \%)\end{array}$ & $\begin{array}{c}2+ \\
\text { (in 5\%-25\%) }\end{array}$ & Poorly differentiated & Focal intercellular bridging & $\mathrm{CK} 7+, \mathrm{p} 63+, \mathrm{TF} 1-$ \\
\hline
\end{tabular}

IHC, immunohistochemistry; CK, cytokeratin; TTF1, thyroid transcription factor 1.

Table 6. Sensitivity and specificity of p40 and p63 in lung squamous cell carcinoma

\begin{tabular}{lccccc}
\hline & Sensitivity (\%) & Specificity (\%) & PPV (\%) & NPV (\%) & p-value \\
\hline p40 & 77.1 & 100 & 100 & 81.4 & $<.001$ \\
p63 & 85.7 & 62.9 & 69.8 & 81.5 & $<.001$ \\
\hline
\end{tabular}

PPV, positive predictive value; NPV, negative predictive value. 
incorrect diagnosis. ${ }^{7,11}$ p63 is a sensitive marker for squamous differentiation, but its specificity is only $60 \%-82 \% .{ }^{9,10}$ Frequent p63 reactivity, particularly in adenocarcinoma and lymphoma, is a significant pitfall in diagnosis of lung cancer.

Accurate histologic diagnosis and subtyping has major therapeutic implications. ${ }^{14}$ Current clinical practice guidelines recommend patients with lung adenocarcinoma or NSCLC favouring adenocarcinoma should undergo molecular testing. ${ }^{15}$ Incorrect diagnosis of squamous cell carcinoma may exclude patients from receiving targeted therapy, or worse, it may endanger patients to complications of the treatment. ${ }^{3,4}$

In studies conducted on mainly resected specimens, $\mathrm{p} 40$ demonstrated 98\%-100\% specificity with comparable sensitivity to that of p63, indicating its reliability for diagnosing squamous cell carcinoma. ${ }^{9,10,12}$ Our study was conducted to investigate the expression of p40 and p63 in predominantly small biopsies and few resected specimens of lung squamous cell carcinoma and adenocarcinoma. The present study was in line with previous reports in concluding that $\mathrm{p} 40$ is more specific than the standard p63 antibody for diagnosing lung squamous cell carcinoma. However, our study demonstrated a lower sensitivity for p40 compared to prior studies.

Based on a literature review, a case with negative $\mathrm{p} 40$ is less likely to be squamous cell carcinoma. A study by Tacha et al. ${ }^{12}$ described six cases of poorly differentiated lung squamous cell carcinoma (5.6\%) that were negative for p40; subsequent staining with other squamous markers were also negative. Kadota et al. ${ }^{5}$ reclassified 31 cases $(6.5 \%)$ of lung tumours that were originally diagnosed as squamous cell carcinoma into other histologic subtypes based on negative p40 staining. Rekhtman et al. ${ }^{16}$ reclassified three tumours with an initial diagnosis of squamous cell carcinoma and lack of p 40 expression as solid adenocarcinoma. In the present study, eight cases originally diagnosed as squamous cell carcinoma were negative for $\mathrm{p} 40$. Based on the review of histology sections of these cases as well as the p40 findings, these cases were reclassified as poorly differentiated or solid type adenocarcinoma. Thus, $\mathrm{p} 40$ is highly recommended in cases of poorly differentiated carcinomas to confirm or exclude the line of squamous differentiation.

Three discrepant cases with p63 positivity, p40 negativity, and TTF1 negativity were initially diagnosed as squamous cell carcinoma. These cases were poorly-differentiated tumours with focal areas displaying squamoid morphology such as intercellular bridging and abundant eosinophilic cytoplasm (Fig. 3). TTF1 is not exhibited in $25 \%$ of lung adenocarcinomas, and the three discrepant cases in this study could be categorized as adenocarcinoma. Since there are few discrepant cases, future studies involving larger sample sizes of poorly differentiated cases are recommended to determine the sensitivity and specificity of p63 and p40 markers in defining subtypes of NSCLC. A threepanel approach of using p63, p40, and TTF1 in cases of carcinoma with complex tumour differentiation would assist in reaching a specific diagnosis.

The majority of cases $(92.9 \%)$ in the current study were small biopsy tissues $(n=65)$ with only five resected specimens (7.1\%). As addressed by Travis et al., small biopsy specimens may not be representative of the total tumour due to histologic heterogeneity of lung cancers. Also, the distinguishable morphologic features of squamous or adenocarcinoma differentiation are only identifiable if the solid tumour component was sampled. ${ }^{17}$

Two p40 antibodies are available for current laboratory use, rabbit polyclonal p40 (RPp40) and mouse monoclonal p40 (MMp40, BC28). RPp40 was used in the above-mentioned studies. ${ }^{5,9,10}$ Nonaka ${ }^{10}$ reported variable background staining that did not hinder the staining interpretation. RPp 40 has been demonstrated to stain macrophages in lung tissue and non-specific cytoplasmic staining in neoplastic cells while MMp40 has clear nuclear staining without non-specific background staining. ${ }^{12}$ Mouse monoclonal antibody was used in the present
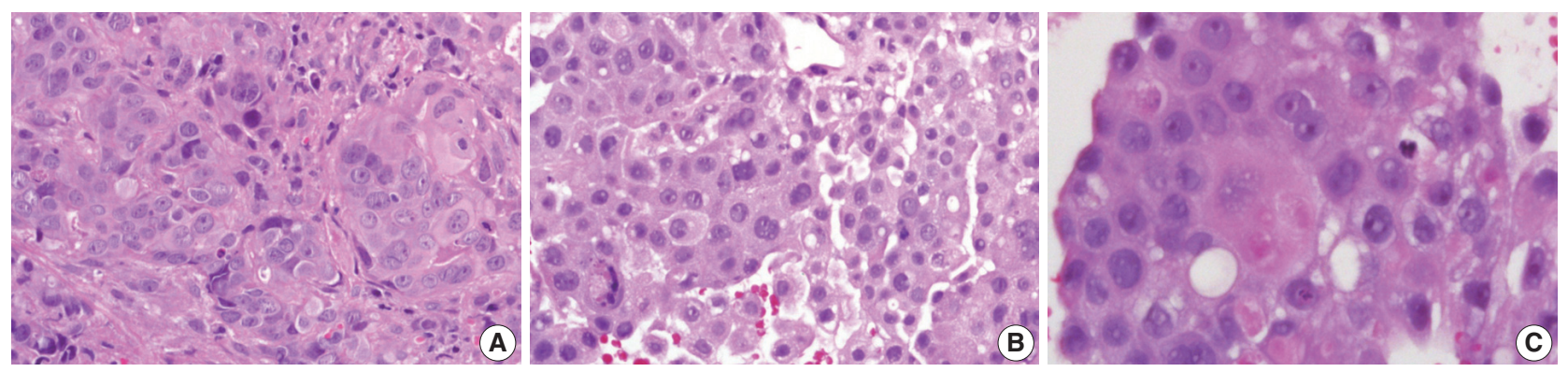

Fig. 3. Poorly differentiated tumour with squamous-like morphology. Tumour cells with abundant eosinophilic cytoplasm and distinct cell borders (A), intercellular bridges (B), and keratinization features (C). 
study, and distinctive nuclear staining with a clean background was observed.

In summary, we demonstrated strong and diffuse $\mathrm{p} 40$ expression in the majority of lung squamous cell carcinomas and absence of p 40 expression in all lung adenocarcinomas. Expression of p63 is similar to that of p40 in lung squamous cell carcinoma, but there was variable p63 immunoreactivity in lung adenocarcinoma. In poorly differentiated cases, a three-panel approach of p63, p40, and TTF-1 may help to distinguish adenocarcinoma from squamous cell carcinoma. Thus, p40 is an excellent marker for distinguishing lung squamous cell carcinoma from adenocarcinoma, and that its expression is equivalent to that of p63 in lung squamous cell carcinoma.

\section{Conflicts of Interest}

No potential conflict of interest relevant to this article was reported.

\section{Acknowledgments}

This study was funded by the Faculty of Medicine, Universiti Kebangsaan Malaysia (project code: FF-2016-077). The authors declare no conflict of interest in the conduct of this study.

\section{REFERENCES}

1. Ferlay J, Soerjomataram I, Dikshit R, et al. Cancer incidence and mortality worldwide: sources, methods and major patterns in GLOBOCAN 2012. Int J Cancer 2015; 136: E359-86.

2. Azizah AM, Nor Saleha IT, Noor Hashimah A, Asmah ZA, Mastulu W. Malaysian National Cancer Registry report 2007-2011. Malaysia cancer statistics, data and figure. Putrajaya: National Cancer Institute, Ministry of Health, 2016.

3. Travis WD, Brambilla E, Noguchi M, et al. Diagnosis of lung cancer in small biopsies and cytology: implications of the 2011 International Association for the Study of Lung Cancer/American Thoracic Society/European Respiratory Society classification. Arch Pathol Lab Med 2013; 137: 668-84.

4. Travis WD, Brambilla E, Burke AP, Marx A, Nicholson AG. WHO classification of tumours of the lung, pleura, thymus and heart. 4th ed. Lyon: International Agency for Research on Cancer, 2015.

5. Kadota K, Nitadori J, Rekhtman N, Jones DR, Adusumilli PS, Travis WD. Reevaluation and reclassification of resected lung carcinomas originally diagnosed as squamous cell carcinoma using immunohistochemical analysis. Am J Surg Pathol 2015; 39: 1170-80.

6. Pelosi G, Fabbri A, Bianchi F, et al. DeltaNp63 (p40) and thyroid transcription factor-1 immunoreactivity on small biopsies or cellblocks for typing non-small cell lung cancer: a novel two-hit, sparing-material approach. J Thorac Oncol 2012; 7: 281-90.

7. Rekhtman N, Ang DC, Sima CS, Travis WD, Moreira AL. Immunohistochemical algorithm for differentiation of lung adenocarcinoma and squamous cell carcinoma based on large series of wholetissue sections with validation in small specimens. Mod Pathol 2011; 24: 1348-59.

8. Nobre AR, Albergaria A, Schmitt F. p40: a p63 isoform useful for lung cancer diagnosis: a review of the physiological and pathological role of p63. Acta Cytol 2013; 57: 1-8.

9. Bishop JA, Teruya-Feldstein J, Westra WH, Pelosi G, Travis WD, Rekhtman N. p40 (DeltaNp63) is superior to p63 for the diagnosis of pulmonary squamous cell carcinoma. Mod Pathol 2012; 25: 40515.

10. Nonaka D. A study of $\Delta \mathrm{Np} 63$ expression in lung non-small cell carcinomas. Am J Surg Pathol 2012; 36: 895-9.

11. Mukhopadhyay S, Katzenstein AL. Subclassification of non-small cell lung carcinomas lacking morphologic differentiation on biopsy specimens: Utility of an immunohistochemical panel containing TTF-1, napsin A, p63, and CK5/6. Am J Surg Pathol 2011; 35: 15-25.

12. Tacha D, Bremer R, Haas T, Qi W. An immunohistochemical analysis of a newly developed, mouse monoclonal p40 (BC28) antibody in lung, bladder, skin, breast, prostate, and head and neck cancers. Arch Pathol Lab Med 2014; 138: 1358-64.

13. Perez-Moreno P, Brambilla E, Thomas R, Soria JC. Squamous cell carcinoma of the lung: molecular subtypes and therapeutic opportunities. Clin Cancer Res 2012; 18: 2443-51.

14. Nicholson AG, Gonzalez D, Shah P, et al. Refining the diagnosis and EGFR status of non-small cell lung carcinoma in biopsy and cytologic material, using a panel of mucin staining, TTF-1, cytokeratin 5/6, and P63, and EGFR mutation analysis. J Thorac Oncol 2010; 5: 436-41.

15. Lindeman NI, Cagle PT, Beasley MB, et al. Molecular testing guideline for selection of lung cancer patients for EGFR and ALK tyrosine kinase inhibitors: guideline from the College of American Pathologists, International Association for the Study of Lung Cancer, and Association for Molecular Pathology. J Thorac Oncol 2013; 8: 823-59.

16. Rekhtman N, Paik PK, Arcila ME, et al. Clarifying the spectrum of driver oncogene mutations in biomarker-verified squamous carcinoma of lung: lack of EGFR/KRAS and presence of PIK3CA/AKT1 mutations. Clin Cancer Res 2012; 18: 1167-76.

17. Travis WD, Rekhtman N, Riley GJ, et al. Pathologic diagnosis of advanced lung cancer based on small biopsies and cytology: a paradigm shift. J Thorac Oncol 2010; 5: 411-4. 